cther than the depth of the humid layer. The width was negligible.

Immediately after formation, the ice crystals began to rise, presumably due to latent heat release. In most cases the humid layer occurred under a temperature inversion and the curtain then rose and flattened out under this within a few minutes. The cloud thus formed would be some 1,000 ft. wide and up to $500 \mathrm{ft}$. deep and would persist until out of sight, not appreciably increasing in volume, but appearing to become denser with time; this suggests considerable growth of the individual crystals at the expense of the surrounding air, made possible by small-scale turbulence at the edges. So far, none of these generated clouds has been observed for more than twenty minutes after seeding.

By using a cloud-droplet eamera in the aircraft, photographs have been obtained of the generated ice crystals. From such photographs it has been possible to make some preliminary studies of the growth of the generated crystals, and to compare them with ice crystals in adjacent natural clouds.

Some preliminary tests on the effect of rate of seeding, of size of 'dry ice' granules, and other seeding variables have also been made, attended, however, with some difficulty, as there is evidence that a considerable and uncontrollable variable may be local variations in humidity, on a scale suggested by the incidence of natural clouds of this type, which are generally very patchy.

As a footnote on the possibilities of interference with Nature, it is interesting to observe that just as success in inducing precipitation seems to require conditions where natural precipitation could well occur anyway, so every time clouds have been generated, natural clouds (or haze) have formed at about the same time and place.

Flight Research Section,

D. Fraser

National Research Council, Arnprior,

Ontario.

March 21.

${ }^{1}$ Schaefer, I. J., Science (Nov. 15, 1946).

${ }^{2}$ First Quarterly Progress Report (March--June, 1947).

\section{Formation of Frozen Rain-drops and the Condition of Ice-crusted Trees}

In districts of high latitudes, it is often found that the central portions of fine suspended water-drops remain unfrozen, even though the temperature of the water is cooled down to $-20^{\circ} \sim-30^{\circ} \mathrm{C}$. This curious phenomenon can be explained by means of my theory of transformation stress; there is an outer spherical crust of ice and an inner water core, the freezing beginning from the outside and proceeding towards the centre. By applying this system of two spheres to the case of the freezing of water, we obtain for the mean intensity of pressure and for the depression of freezing point the following values:

$$
0.355 K \frac{\delta v}{v}=\vec{p}=3,280 \text { atmos. }
$$

and

$$
\Delta T=0 \cdot 355 K T\left(\frac{\delta v}{v}\right)^{2} / \rho \lambda=-26 \cdot 4^{\circ} \mathrm{C} .
$$

In these expressions for $\bar{p}$ and $\Delta T, K=1 \cdot 03 \times 10^{11}$,
$T=273, \rho=0.91, \lambda=80 \times 4.2 \times 10^{7}, \frac{\delta v}{v}=0.09$ (to be published in the near future). The observed value of $\Delta T$ is $-33^{\circ} \mathrm{C}$., in fair agreement with the theoretical value. Thus, during freezing, the surface of the inner sphere of water may sustain a mean pressure of 3,280 atmospheres at $-33^{\circ} \mathrm{C}$., and there. fore below this temperature the water remains as such. This provides an explanation of frozen raindrops.

Next, take the case of ice-crusted trees. The water penetrates into the inner portions of tree branches, leaves, etc., and freezes into ice; but the water in the central portion remains as such, owing to the high pressure of the surrounding ice. Hence, even in very cold weather, the translocation of the necessary water from roots to leaves of these trees is not very much prevented, and so the damage is not very great.

Otaku, Denenchofu 3-596,

Kotaro Honda

Tokyo.

Dec. 26

\section{Biosynthesis of 'Asymmetric' Citric Acid : a Substantiation of the Ogston Concept}

A. G. OGSToN ${ }^{1}$, in a recent note in Nature on the "Interpretation of Experiments on Metabolic Processes, using Isotopic Tracer Elements", introduced the important concept that an enzyme might be able to distinguish between the chemically 'identical' groups of a symmetrical compound. Ogston pointed to the dangers of the contrary assumption, and as one example he questioned the conclusion that citric acid was not an intermediate in the Krebs cycle of oxidations in so far as this conclusion was based upon the finding that the fixation of isotopically labelled carbon dioxide led to the formation of asymmetrically labelled $\alpha$-keto-glutaric acid. It had been assumed ${ }^{2,3,4}$ that if citric acid were on the pathway leading to $\alpha$-ketoglutaric acid, the latter would have had isotopic carbon equally distributed between the two carboxylic acid groups.

We have tested the Ogston concept as it applies to this case. Carbon dioxide labelled with carbon-14 was released into the gas phase of Warburg flasks containing a reaction mixture in which a rat-liver homogenate was oxidizing oxalacetate and pyruvate under conditions that lead to the accumulation of citrate $^{5,6}$. Citric acid containing carbon-14 was isolated using a silica gel column according to the method of Isherwood ${ }^{7}$ as employed by Stutz and Burris, of the Biochemistry Department at this University. The citric acid (a structurally symmetrical molecule) was enzymatically converted to $\alpha$-ketoglutaric acid in the presence of $0.0003 M$ arsenite by means of a preparation derived from a rat-liver homogenate ${ }^{8}$. The $\alpha$-ketoglutaric acid was converted to the 2,4-dinitrophenylhydrazone, and this was purified by passage through a column of diatomaceous earth according to the method of LePage ${ }^{9}$ to separate it from any possible pyruvate and oxalacetate hydrazones. It was then mixed with pure nonradioactive $\alpha$-ketoglutarate dinitrophenylhydrazone and recrystallized five times. The product was oxidized to succinic acid and carbon dioxide using potassium permanganate as described by Krebs ${ }^{10}$, and carrier succinic acid was added to the reaction mixture and isolated and purified by recrystallization. 